\title{
REVIEW THE DEEP LEARNING TECHNIQUE FOR MISSING DATA CLASSIFICATION IN IOT APPLICATIONS FOR NETWORK PERFORMANCE IMPROVEMENT
}

\author{
Gopal Patil ${ }^{1}$, Dr. Raj Thaneeghaivel V ${ }^{2}$ \\ ${ }^{1}$ Ph.D. Research Scholar, Department of Computer Science and Application, Sarvepalli Radhakrishnan University, NH 12, RKDF \\ IST Campus, Hoshangabad Road, Misrod, Bhopal (M.P.) \\ ${ }^{2}$ Associate Professor, Department of Computer Science and Application, Sarvepalli Radhakrishnan University, NH 12, RKDF IST \\ Campus, Hoshangabad Road, Misrod, Bhopal (M.P.).
}

\begin{abstract}
In order to ensure product safety and increase production quality, The construction of mine Internet of Things networks continues to accelerate mining enterprises. Given the large increase in the number of networked devices connectivity capability in the mine, there is considerable strain on the mine network communication facilities. We suggest an Innovative Solution Using Deep Learning for Missing Data Classification in IoT Network Performance Enhancement System Market Classifier based on neural networks to improve the quality of service in the connectivity infrastructure of mine networks. The classifier uses a transformation wavelet to delete the data flow and to build compliance characteristics to identify the market categories of the system.Owing to the findings of the classification, the system changes the specifications of the network services given to the terminal equipment in a versatile manner. In this way, the system's network capacity can be fairly distributed. We assess the output of the classifier model using the test data collection. We review the deep learning technique in IoT applications for Network Improvement for missing data classification..
\end{abstract}

Keywords: Cross Neural Network, IoT applications , Internet of Things, Missing values.

\section{Introduction}

In the current situation, sensors of wearable is highly important of the health field and science application[1]. The current market for sport and sport logging, regulation, human user contact, care, patient tracking for Ambient Living Purposes has restricted the scale and costs of some of the wearable monitors[2]. Significant improvement may be achieved, since this is from IoT which does not require steps to secure people on the other hand. [3]. The wearable tracker have generated a lot of media noise attributable to weight, blood pressure, calories, and movement, some features lack the capacity to distinguish or understand the distinction between medical grade monitoring and adequate knowledge recording. [4]. On a positive note, IoT has recently announced its program wellness, which will act as a Fitness Kit Creation Platform to Health data and other applications aggregate [5]. Software developers may use various types of sensing techniques. They gather data from both wired and wireless sensors and other devices. [6] The Data Management Software has verified the security and heath data in the storage of cloud, and users of the service will be able to helathcare give this information, doctors, hospitals and relatives if required (Figure 1). Advances in networking, microelectronics, sensor production and data extraction have opened up new possibilities for wearable health platforms[8]. It is actually capable of implementing remote area[9]. In the past, sensors and electronics have become too complicated to use in lightweight, sensor-scale, wearable systems. Medical providers do not need a wireless tracker or an app to download to ensure that a marathon runner performs effectively to monitor the cost of calories on a day-to-day basis. [10].

Conversely, a wireless medical device used to provide a lifethreatening warning to create a degree of certainty could be needed[11]. In the world population, there have been several demographic changes, especially in the West, which has contributed to the need for important sign monitoring[12]. Population ageing and increased obesity are two big trends. These two factors raise the probability that different diseases will need substantial medical attention and, therefore, significant costs[14]. Globally, accept this and strive to offer healthcare care more efficiently across personal health networks and telemedicine. [15] The following paper is structured as follows, as defined in Section II of Extensive study on the Health Care Services wireless sensor network, and in Section III of the background study the proposed structure is discussed below. Section inishes with Final \& Future Work. 


\section{Related Work}

P. Li, Z. Chen et al[1] A tensor convolution operation is developed to avoid over-fitting and improve training efficiency in order to make the most of the local features and topologies found in the Big Data. In addition, a high-order backpropagation algorithm is proposed in high-order space to train the parameters of a profound convergence model of computation.

S. Huang et al.,[2] This article suggests a current Gated Residual Neural Network (GrrNet) for the AMC, With signal amplitude and phase received by GrrNet inputs. GrrNet primarily configures the ResNet Extractor module to remove a highly representative feature and then extracts temporary information for arbitrary time-duration modulation from the corresponding GRU module.

T. Jan et al[3] In this article, an Automation and Compact Machine Learning Model was developed to classify IoT data in a heterogeneous and complex IoT context called the Adaboted Probabilistic Neural Network (PNN). Classified IoT programmes can be used in order to prioritise capital distribution to fund critical services. The model suggested is focused on the classification of IoT resources and shows comparable classification accuracy with respect to other stateof-the-art methods, albeit at low computational costs.

F. Wang et al[4] As for the slow, rapid collapse of the local minimum value preventing BP's use of the neural network in the Internet of Things (IOT), the standard BP neural network provides an optimised BP algorithm with a variable learning rate. The paper uses the neural network toolkit and GUI

Table 1: Missing Data Classification in IoT Applications

\begin{tabular}{|c|c|c|c|}
\hline Author & Algorithm & Advantage & Research Gaps \\
\hline P. Li, et al[1] & $\begin{array}{l}\text { Deep } \\
\text { convolutional } \\
\text { computation } \\
\text { model } \\
\end{array}$ & higher classification accuracy & Need to Improvement \\
\hline S. Huang et al[2] & $\begin{array}{l}\text { novel gated } \\
\text { recurrent } \\
\text { residual } \\
\text { neural } \\
\text { network } \\
\text { (GrrNet) } \\
\end{array}$ & influence of the network parameters & Need to Improvement \\
\hline T. Jan et al[3] & $\begin{array}{l}\text { probabilistic } \\
\text { neural } \\
\text { network } \\
(\mathrm{PNN})\end{array}$ & reduced computational cost & Work $\mathrm{n}$ less datasets \\
\hline F. Wang et al[4] & BP algorithm & better data classification performance & $\begin{array}{l}\text { Work on slow convergence Need } \\
\text { to Improvement }\end{array}$ \\
\hline $\begin{array}{l}\text { M. } \quad \text { Dawodi,et } \\
\text { al[5] }\end{array}$ & $\begin{array}{l}\text { Convolutiona } \\
1 \quad \text { Neural } \\
\text { Network } \\
(\mathrm{CNN}) \\
\end{array}$ & high accuracy & $\begin{array}{l}\text { model achieved } 88.2 \% \text { Need to } \\
\text { Improvement }\end{array}$ \\
\hline
\end{tabular}

\section{Missing Data Classification in IoT Applications}

The study lays out an innovative IoT system for the long-term, individual tracking of a person's activities. The IBCN suggests sensor of wearable with various kinds of human behavior uncertainties. The system incorporates a wearable sensor and interfaces to distinguish data experiment sets that rely on the MATLAB environment to verify the efficiency of the improved algorithm. The experimental results suggest that the algorithm has improved the efficiency of the classification of data.

M. Dawodi et al[5] This set uses our audio files as a database, as Dari language databases were not available on the market at the time. This paper uses CNN to detect independent words in Dari automatically. This paper is also available online. In addition, during preparation, mel frequency coefficients (MFCC) are used to learn how to represent the functions.

P. Hattikatti,[6] The suggested solution involves the usage of $\mathrm{CNN}$ as a 7-layer local binary pattern (LBP). Classification suggests that CNNs can determine pulmonary trends. The classification has been carried out. The CT Images analysis for this observational research has been legally checked by a licenced radiologist.

T. S. Fatayer et al[7] We need safe and strong technical results to identify these attacks. This post contains a number of attacks that IoT contacts are undergoing using Classification Algorithms from Artificial Neural Networks (ANN). These are all LVQ and Radial Base Function (RBB) and multilayer perceptron variations. Multilayer Perceptron (MLP).
Deep Learning technology to include information on a variety of behavioural deduction behaviours. The proposed approach is characterized by Data dependence and model-dependent certainty laboratory analysis. The approach suggested was meant to be extended to applications that would have personalized information with a wide variety of wearable 
sensors, The measurement of various types of uncertainties is important for the creation of active learning paradigms and novelty identification and for the detection of errors and data fusion that are both relevant to the production of effective and personalized market recognition systems. The infrastructure configuration incorporates $\mathrm{Wi}-\mathrm{Fi}$ and Cloud on-board software so that the network can be constantly updated with new training sets while introducing customers. This alternative solution can be used to build light-weight, compact, autonomous integrated human recognition systems by using ICBN technology to integrate complex signal processing systems. Preprocessing data for data processing research using DBN algorithms is one of the most critical steps. Realistic implementation increases the accuracy of the learning functionality by utilising DBN algorithms for learning data functions, pre-processing the initial functional data and then executing the feature learning phase. The first move is to normalise the data function. There are several approaches to normalize data, and the particular standardization approach typically selects various standardization methods depending on the specific implementation context of the data., there are a variety of the main algorithm for standardizing function data:

(1) Simple scaling: The goal is to ensure that the values of the data features fall within the spectrum of $[0,1]$ or $[-1,1]$ after the standardisation process has transformed the data features of each data dimension the many data is main for data mining, since certain algorithms, including epsilon, are common for feature data and are scaled at a given interval in the default whitening of Pca.

(2) Minimum-maximum normalization: The minimal limit normalisation is the basic linear scaling. The minimum maximum one standardisation shall be determined where the the dataset value of the min and max However, there are still some drawbacks of this structured algorithm. When new data is available, it could be appropriate to re-discover the of the value min and max recalculate the changes to the value of the maximum and minimum. a straightforward, The continuity between converted and original data is therefore retained.

$$
v^{\prime}=\frac{v-\min }{\max -\min }
$$

(3) When max is the value of the first data set and min is the lowest value of the original data set. Where, there are still some drawbacks of this structured algorithm. When new data is available, it could be appropriate to re-discover the maximum and minimum values and then recalculate the changes to the maximum and minimum values.

(4) Feature Standardization: The aim is to the mean and a variance of units of the value dimension. Standardization feature.This standardization approach is commonly used in data and pre-processing. In the value od actual measurement, the thorough standardization method is dimension by the data norm. The basic method of estimation is as follows

$$
v^{\prime}=\frac{v-u}{\sigma_{A}}
$$

The mean and default deviations are $\mathrm{u}$ and the $\mathrm{z}$-score standardisation algorithm is used if the maximum and minimum A attribute values of the data set is correctly reached or if the data noise is too high to surpass the typical value. The data input is summed first for the PCA/ZCA blanking application. If $x(i)$ is a data set function, the method of zeroaveraging.

$$
\frac{1}{m} \sum_{i} x^{(i)}=0
$$

The secret to the consistency of function learning is data preprocessing. Also in basic feature learning algorithms, it is appropriate for data preprocessing approaches to produce better outcomes. In the function representation of study data in the learning feature network, the initial data collection after data preprocessing can be entered. Convolutionary neural networks are a type of deep artificial neural network, since their dominance. This suggests that it is more analogous to the actual operational theory of the biological neural network, The conventional machine learning algorithm can be avoided by this working mechanism. The method requires tedious learning of functions and data reconstruction. a structured time domain signal are usually obtained from a contiguous data collection. Health status assessment. The physiological timing of big data is focused on the features of large data learning data. In this paper we usage of convolutionary neural network strategies to automatically learn in an unsupervised learning way the deep feature, then used for health status evaluation. Network depth improves, its simulation ability can be vastly increased, more abstract data function representation can be derived within the data, and the overall classification output can be further strengthened based on various network structure DBN. However, ANN has the highest results on Acc relative to the three, but there is a small scarcity of Spec Sen and Mse. The findings obtained when adding multiple deep learning models to NTC are discussed in this section. In particular, the effect of many critical hyper-parameters and design decisions is analyzed: the model architecture, the chosen features and the number of packets derived from the flows of the network. It has been observed that CDBN's efficiency is higher to the CNN architucture. The efficiency of CNN is higher than DBN, because with the rise in the image on each classification index essentially improves. Improvements have also demonstrated that the DBN architecture is strongly dependent on samples from big data preparation. For this reason, each classification index is between 4000 and 500, test image and number of training image will be of considerable significance.The amount of images is often due to limitations in the experimental conditions of the hardware. One of the reasons for this is that CDBN can help classify the features in the lung picture due to the improved convolution activity and coarse 
regularization in the $\mathrm{CDBN}$ experiment. In the assessment phase of this article, the focus of this paper is feature learning. Then, to determine the health condition, In the data collection, the model evaluated eight physiological signals. Experimental findings show that a high-level algorithm can learn from initial physiological evidence is the feature extraction approach used in this article. A feasible algorithm is also used to determine health status via the Gaussian Multivariate Distribution Theory. Three forms of medical DBN multimedia database MIAS have been studied, in conjunction with DBN, in a comparative analysis of a deep conviction network, an automatic stack encoder and a convolutionary neural network. The benefits and disadvantages of the three forms of networks are evaluated. Secondly, the principle of automated coding in the training of convolutionary neural networks is used in the training network, This basically the issue of the need to optimise the network with tagged information for a traditionally complicated neural network. To shape a health scenario, this paper uses and uses Gaussian function to calculate networking probability values to create a health model that focuses on a variety of job algorithms. The experimental findings indicate that the approach used in this paper is an efficient technique which can also be applied to other data forms. CNN use in DBN is the approach used in this article. Although this paper cites several other DBN algorithms, the algorithm theory is also mentioned, but, for example, there is no other deep trust network.

\section{Conclusion}

The proposed approach is stable and offers outstanding F1 scores with more than 100 different classification labels in extremely unbalanced data sets. It operates with very few features and does not require any feature engineering. We used high-level packet-based header-based data for model training. Payload or IP e-mail, which are possibly sensitive or encrypted, need not be relied on. A basic RNN model has already produced very successful outcomes, but when the RNN model matches the previous CNN, it is important to note that these results are enhanced. To be able to boost performance We consider experimenting with new $\mathrm{CNN}$ and LSTM models implementations and variants as a potential job, and thus CNNs are a valid candidate for operating with the same type of vector time series.in near future try to implement realistic environment.

\section{References}

[1] P. Li, Z. Chen, L. T. Yang, Q. Zhang and M. J. Deen, "Deep Convolutional Computation Model for Feature Learning on Big Data in Internet of Things," in IEEE Transactions on Industrial Informatics, vol. 14, no. 2, pp. 790-798, Feb. 2018, doi: 10.1109/TII.2017.2739340.
[2] S. Huang et al., "Automatic Modulation Classification Using Gated Recurrent Residual Network," in IEEE Internet of Things Journal, vol. 7, no. 8, pp. 7795-7807, Aug. 2020, doi: 10.1109/JIOT.2020.2991052.

[3] T. Jan and A. S. M. Sajeev, "Boosted Probabilistic Neural Network for IoT Data Classification," 2018 IEEE 16th Intl Conf on Dependable, Autonomic and Secure Computing, 16th Intl Conf on Pervasive Intelligence and Computing, 4th Intl Conf on Big Data Intelligence and Computing and Cyber Science and Technology Congress(DASC/PiCom/DataCom/CyberSciTech),

Athens, 2018, pp. 408-411, doi: 10.1109/DASC/PiCom/DataCom/CyberSciTec.201 8.00082 .

[4] F. Wang and L. Niu, "An improved BP neural network in Internet of Things data classification application research," 2016 IEEE Information Technology, Networking, Electronic and Automation Control Conference, Chongqing, 2016, pp. 805-808, doi: 10.1109/ITNEC.2016.7560472.

[5] M. Dawodi, J. A. Baktash, T. Wada, N. Alam and M. Z. Joya, "Dari Speech Classification Using Deep Convolutional Neural Network," 2020 IEEE International IOT, Electronics and Mechatronics Conference (IEMTRONICS), Vancouver, BC, Canada, 2020, pp. 1-4, doi: 10.1109/IEMTRONICS51293.2020.9216370.

[6] P. Hattikatti, "Texture based interstitial lung disease detection using convolutional neural network," 2017 International Conference on Big Data, IoT and Data Science (BID), Pune, 2017, pp. 18-22, doi: 10.1109/BID.2017.8336567.

[7] T. S. Fatayer and M. N. Azara, "IoT Secure Communication using ANN Classification Algorithms," 2019 International Conference on Promising Electronic Technologies (ICPET), Gaza City, Palestine, 2019, pp. 142-146, doi: 10.1109/ICPET.2019.00033.

[8] N. Mohanasundaram, "Recurrent Neural Network approach for data prediction of a chemical process plant," 2020 Fourth International Conference on ISMAC (IoT in Social, Mobile, Analytics and Cloud) (I-SMAC), Palladam, India, 2020, pp. 1257 1261, doi: 10.1109/I-SMAC49090.2020.9243465.

[9] R. Dandavate and V. Patodkar, "CNN and Data Augmentation Based Fruit Classification Model," 2020 Fourth International Conference on I-SMAC (IoT in Social, Mobile, Analytics and Cloud) (ISMAC), Palladam, India, 2020, pp. 784-787, doi: 10.1109/I-SMAC49090.2020.9243440. 
[10] Rajawat A.S., Upadhyay P., Upadhyay A. (2021) Novel Deep Learning Model for Uncertainty Prediction in Mobile Computing. In: Arai K., Kapoor S., Bhatia R. (eds) Intelligent Systems and Applications. IntelliSys 2020. Advances in Intelligent Systems and Computing, vol 1250. Springer, Cham. https://doi.org/10.1007/978-3-03055180-3_49.

[11] A. Saini, T. Gupta, R. Kumar, A. K. Gupta, M. Panwar and A. Mittal, "Image based Indian monument recognition using convoluted neural networks," 2017 International Conference on Big Data, IoT and Data Science (BID), Pune, 2017, pp. 138-142, doi: 10.1109/BID.2017.8336587.

[12] F. Hamami and I. Fithriyah, "Classification of Air Pollution Levels using Artificial Neural Network," 2020 International Conference on Information Technology Systems and Innovation (ICITSI), Bandung - Padang, Indonesia, 2020, pp. 217-220, doi: 10.1109/ICITSI50517.2020.9264910.

[13] M. Kwon, H. You, J. Kim and S. Choi, "Classification of Various Daily Activities using Convolution Neural Network and Smartwatch," 2018 IEEE International Conference on Big Data (Big Data), Seattle, WA, USA, 2018, pp. 49484951, doi: 10.1109/BigData.2018.8621893.

[14] N. Al-Milli and W. Almobaideen, "Hybrid Neural Network to Impute Missing Data for IoT Applications," 2019 IEEE Jordan International Joint Conference on Electrical Engineering and Information Technology (JEEIT), Amman, Jordan, 2019, pp. 121-125, doi: 10.1109/JEEIT.2019.8717523.

[15] H. Nishizaki and K. Makino, "Signal Classification Using Deep Learning," 2019 IEEE International Conference on Sensors and Nanotechnology, Penang, Malaysia, 2019, pp. 1-4, doi: 10.1109/SENSORSNANO44414.2019.8940077.

[16] M. Pamukov, V. Poulkov and V. Shterev, "NSNN Algorithm Performance with Different Neural Network Architectures," 2020 43rd International Conference on Telecommunications and Signal Processing (TSP), Milan, Italy, 2020, pp. 280-284, doi: 10.1109/TSP49548.2020.9163469.

[17] A. Singh Rajawat and S. Jain, "Fusion Deep Learning Based on Back Propagation Neural Network for Personalization," 2nd International Conference on Data, Engineering and Applications (IDEA), Bhopal, India, 2020, pp. 1-7, doi: 10.1109/IDEA49133.2020.9170693.

[18] R. Adeogun, I. Rodriguez, M. Razzaghpour, G. Berardinelli, P. H. Christensen and P. E. Mogensen,
"Indoor Occupancy Detection and Estimation using Machine Learning and Measurements from an IoT LoRa-based Monitoring System," 2019 Global IoT Summit (GIoTS), Aarhus, Denmark, 2019, pp. 1-5, doi: 10.1109/GIOTS.2019.8766374.

[19] P. S. Patil and N. V. Dharwadkar, "Analysis of banking data using machine learning," 2017 International Conference on I-SMAC (IoT in Social, Mobile, Analytics and Cloud) (I-SMAC), Palladam, 2017, pp. 876-881, doi: 10.1109/ISMAC.2017.8058305.

[20] A. S. Rajawat, O. Mohammed and P. Bedi, "FDLM: Fusion Deep Learning Model for Classifying Obstructive Sleep Apnea and Type 2 Diabetes," 2020 Fourth International Conference on I-SMAC (IoT in Social, Mobile, Analytics and Cloud) (ISMAC), Palladam, India, 2020, pp. 835-839, doi: 10.1109/I-SMAC49090.2020.9243553.

[21] S. Mittal and S. Mittal, "Indian Banknote Recognition using Convolutional Neural Network," 2018 3rd International Conference On Internet of Things: Smart Innovation and Usages (IoT-SIU), Bhimtal, 2018, pp. 1-6, doi: 10.1109/IoTSIU.2018.8519888. 